Théologiques

Théologiques

\title{
Les choix apostoliques de Paul et leur portée
}

Ou : comment être témoin d'une vérité divine sans devenir un fanatique religieux?

\section{Jean-Michel Poffet}

Volume 12, numéro 1-2, 2004

Le Soi dans tous ses états

URI : https://id.erudit.org/iderudit/011564ar

DOI : https://doi.org/10.7202/011564ar

Aller au sommaire du numéro

\section{Éditeur(s)}

Faculté de théologie et de sciences des religions, Université de Montréal

\section{ISSN}

1188-7109 (imprimé)

1492-1413 (numérique)

Découvrir la revue

Citer cet article

Poffet, J-M. (2004). Les choix apostoliques de Paul et leur portée : ou : comment être témoin d'une vérité divine sans devenir un fanatique religieux ?

Théologiques, 12(1-2), 233-244. https://doi.org/10.7202/011564ar

Tous droits réservés (C) Faculté de théologie et de sciences des religions, Université de Montréal, 2005
Ce document est protégé par la loi sur le droit d'auteur. L'utilisation des services d'Érudit (y compris la reproduction) est assujettie à sa politique d'utilisation que vous pouvez consulter en ligne.

https://apropos.erudit.org/fr/usagers/politique-dutilisation/ 


\section{Les choix apostoliques de Paul et leur portée ${ }^{1}$}

Ou : comment être témoin d'une vérité divine sans devenir un fanatique religieux ?

Jean-Michel Poffet, o.p.

Directeur

École biblique de Jérusalem

\section{Introduction}

Parler de Dieu n'est pas sans risque... Prétendre être témoin de Dieu en ce monde est non seulement une entreprise difficile, mais elle éveille dorénavant le soupçon. Le poids de l'autorité divine a trop souvent été invoqué pour cautionner des vues trop humaines, des politiques qui n'étaient pas que spirituelles! La méfiance est devenue si grande qu'en Europe par exemple il n'a pas été possible de mettre le nom de Dieu et de parler de l'apport des religions chrétienne, juive, musulmane, dans le préambule de la Constitution Européenne en préparation. La France en particulier a été la plus réticente, à cause de sa tradition de laïcité, et cela malgré les protestations du Saint Siège ou de la Fédération protestante de France. «L'enjeu, écrivait un journaliste en marge de ces débats, en est la perception des religions, à l'aube d'un $\mathrm{XxI}^{\mathrm{e}}$ siècle où elles sont le plus souvent identifiées aux guerres et aux tensions du monde. [...] La place de la religion dans la sphère publique est à nouveau en débat. Elle est liée aux appréhensions que suscite la montée des sectarismes religieux et des communautarismes. L'invocation permanente du nom de Dieu dans les conflits, en Irlande, au Kosovo, en Afghanistan, en Irak, au Cachemire, finit par décourager même les agnostiques les plus ouverts à l'idée de transcendance. » Les religions monothéistes notamment auraient été à la

1. Texte d'une conférence organisée par la Faculté de théologie et de sciences des religions de l'Université de Montréal et l'Institut de pastorale des Dominicains, prononcée le 28 octobre 2003. 
source de trop de violence au cours de l'histoire. Une historienne, opposée à la mention de Dieu dans cette constitution, écrit: "On serait en droit d'attendre davantage de conscience historique de la part de partis et de personnalités, notamment allemands et espagnols, si prompts à dénoncer les crimes du communisme, mais manifestement amnésiques quant aux persécutions et massacres qui ont décimé les minorités depuis des siècles sur notre continent et au-delà, au nom de convictions et d'une mission condamnant les infidèles. » Mais observons que l'hindouisme par exemple connaît aussi aujourd'hui une phase de violence, et que les régimes athées n'ont pas failli en la matière... (Russie et Chine). Il faudrait évidemment mentionner aussi l'utilisation du nom de Dieu dans certains milieux gouvernementaux aux États-Unis, comme aussi dans les milieux extrémistes musulmans ou chez les colons juifs, voire dans les milieux sionistes. Mais il est plus difficile d'excuser la violence au service de la vérité quand on se veut témoin d'un Dieu d'amour, du Dieu de Jésus-Christ.

Ma problématique est ainsi esquissée: est-il possible d'invoquer une vérité divine et d'apparaître comme "citoyen » du monde ? Est-il possible de tenir ensemble le service de la vérité et la bonté, pour le dire dans des termes bibliques ? Certains, sous prétexte de fidélité, deviennent raides et sans bonté; d'autres, sous prétexte de bonté, finissent par être gravement infidèles à leur foi. En fait, en hébreu, le terme de hèsèd dit à la fois la bonté et la fidélité. Fidélité de la part de Dieu qui s'engage dans l'Alliance et qui appelle à la loyauté et à la fidélité; amour prévenant d'un Dieu prompt au pardon et lent à la colère.

La réponse que je vais esquisser ici relève d'une lecture de la trajectoire parcourue par l'apôtre Paul, lui que tout le monde accuse d'un autoritarisme difficile à supporter. Je soulignerai plus particulièrement l'apport de la première lettre aux Thessaloniciens. Cette lettre avait été négligée pendant longtemps: elle ne fait pas partie des grandes épîtres (Galates, Romains, Corinthiens), on n'y trouve pas les grands thèmes de la théologie paulinienne: grâce, péché, liberté. Cette lettre semblait n'intéresser que par son insistance sur l'eschatologie. Et voilà que depuis quelques années il y a un renouveau d'intérêt pour les épîtres aux Thessaloniciens, et en particulier pour l'attitude humaine et pastorale de l'apôtre. C'est précisément sur ce point que je voudrais m'arrêter ici: l'importance de l'attitude pastorale de Paul, fondée sur une perception profonde du mystère du Christ. Une attitude pastorale étroitement liée à sa façon d'entrevoir le service de la vérité. 


\section{Une fidélité absolue à l'évangile de la grâce}

Paul, sur le chemin de Damas, devient en un instant chrétien et apôtre, du fait de sa rencontre avec le Christ. Il est soudain mis en présence du Christ crucifié et ressuscité. L'apôtre se trouve comme arraché de cette terre, transporté dans les cieux et au moment du jugement. Il était un persécuteur de ce Jésus de Nazareth, de cet homme qui avait des prétentions messianiques et quasi-divines alors qu'il était mort en croix... Après sa rencontre avec le Christ, Paul se trouve apôtre de ce même Jésus Christ et dans cette même communauté chrétienne qu'il persécutait:

Vous avez certes entendu parler de ma conduite jadis dans le judaïsme, de la persécution effrénée que je menais contre l'Église de Dieu et des ravages que je lui causais et de mes progrès dans le judaïsme, où je surpassais bien des compatriotes de mon âge, en partisan acharné des traditions de mes pères. Mais quand Celui qui dès le sein maternel m'a mis à part et appelé par sa grâce daigna révéler en moi son Fils pour que je l'annonce parmi les païens, aussitôt, sans consulter la chair et le sang, sans monter à Jérusalem trouver les apôtres mes prédécesseurs, je m'en allai en Arabie, puis je revins encore à Damas. (Ga 1,13-17)

Paul se découvre objet de l'eudokia (la bienveillance) du Père dans l'acte même de révélation. Il devient apôtre non de par l'initiative du Jésus terrestre mais de par l'initiative du ressuscité. Cette révélation fait de lui et jusqu'à sa mort un témoin de Jésus. Cette thématique (révélation du Fils et eudokia du Père), nous la trouvons au baptême de Jésus et à la transfiguration - «Celui-ci est mon Fils bien-aimé, qui a toute ma faveur» (Mt 3,17) - avec pour la transfiguration un ajout — «écoutezle!» —, vu la proximité de la passion et le danger de ne plus voir dans l'ami et le maître crucifié le Fils bien-aimé du Père. On trouve encore la même thématique dans l'hymne de jubilation où la révélation du Fils par le Père est en tension avec une autre logique qui est celle des sages et des intelligents :

En ce temps-là Jésus prit la parole et dit: «Je te bénis, Père, Seigneur du ciel et de la terre, d'avoir caché cela aux sages et aux intelligents et de l'avoir révélé aux tout-petits. Oui, Père, car tel a été ton bon plaisir. Tout m'a été remis par mon Père, et nul ne connaît le Fils si ce n'est le Père, et nul ne connaît le Père si ce n'est le Fils, et celui à qui le Fils veut bien le révéler. » Venez à moi, vous tous qui peinez et ployez sous le fardeau, et moi je vous soulagerai. Chargez-vous de mon joug et mettez-vous à mon 
école, car je suis doux et humble de coeur, et vous trouverez soulagement pour vos âmes. Oui, mon joug est aisé et mon fardeau léger. (Mt 11,2527)

Dans la confession de Pierre enfin, on retrouve ce motif révélation / bienveillance, mais cette fois en tension avec "la chair et le sang», une autre manière de parler de l'homme laissé à ses propres perceptions naturelles: «Bienheureux es-tu Simon, Fils de Jonas, car ce ne sont pas la chair et le sang qui t'ont révélé cela, mais mon Père qui est dans les cieux. Amen, amen, je te le dis: tu es Pierre, et sur cette pierre je bâtirai mon Église. » (Mt 16,17-18)

Une question se pose: la parole de Jésus à Pierre rapportée par Matthieu est-elle une réaction au privilège de Paul, ou bien Paul connaissait-il déjà la tradition qui est à l'arrière-plan de l'évangile de Matthieu? Quoi qu'il en soit, ce qui fonde la grâce d'un apostolat de premier plan et de première responsabilité, c'est cette grâce d'une révélation du Fils non pas seulement à Paul mais EN lui comme l'avait bien noté Jean Chrysostome. A partir de ce moment, Paul devient non seulement apôtre, mais un apôtre d'une fidélité absolue à cette grâce. Il sait mieux que tous les autres que cette confession du Christ ne vient pas de lui, de son initiative, de sa compétence. Sa formation, son tempérament, son histoire ne le préparaient pas à cela... Rappelons le début de l'épître aux Galates: «Paul, apôtre, non de la part des hommes, ni par l'intermédiaire d'un homme, mais par Jésus Christ et Dieu le Père qui l'a ressuscité des morts. » (Ga 1,1) C'est de cette conviction que naît sa fidélité mais aussi son intransigeance:

Je m'étonne que si vite vous abandonniez Celui qui vous a appelés par la grâce du Christ, pour passer à un second évangile - non qu'il y en ait deux; il y a seulement des gens en train de jeter le trouble parmi vous et qui veulent bouleverser l'Évangile du Christ. Eh bien! Si nous-mêmes, si un ange venu du ciel vous annonçait un évangile différent de celui que nous vous avons prêché, qu'il soit anathème! Nous l'avons déjà dit, et aujourd'hui je le répète: si quelqu'un vous annonce un évangile différent de celui que vous avez reçu, qu'il soit anathème! En tout cas, maintenant est-ce la faveur des hommes, ou celle de Dieu que je veux gagner? Est-ce que je cherche à plaire à des hommes? Si je voulais encore plaire à des hommes, je ne serais plus le serviteur du Christ. (Ga 1,6-10)

Ne pas plaire aux hommes, être et demeurer serviteur du Christ: nous avons là le premier aspect de la problématique de l'Apôtre. Une 
problématique de fidélité exigeante: mais comment, dans ces conditions, s'approcher des hommes, être reçu par eux? Non pour s'assimiler à eux mais pour les rencontrer et les gagner à l'évangile? L'argument d'autorité à lui seul suffit-il ? Nous savons, en particulier dans nos sociétés, qu'il est devenu très faible depuis que les Lumières ont installé la raison comme instance première et souvent dernière dans la réflexion. Et quand l'Église, les évêques ou le pape revendiquent le droit et le devoir de parler au nom d'une révélation, d'une vérité révélée, ils renforcent encore ce mécanisme de rejet, ou au moins de scepticisme. La vérité chrétienne est UNE vérité parmi d'autres. C'est la prétention à l'exclusivité qui paraît difficile à admettre. Déjà Paul se trouvait confronté au syncrétisme des Corinthiens, il savait qu'en parlant selon ses convictions, il allait leur paraître insensé:

Oh! Si vous pouviez supporter que je fasse un peu l'insensé! Mais, bien sûr, vous me supportez. J'éprouve à votre égard en effet une jalousie divine; car je vous ai fiancés à un époux unique, comme une vierge pure à présenter au Christ. Mais j'ai bien peur qu'à l'exemple d'Ève, que le serpent a dupée par son astuce, vos pensées ne se corrompent en s'écartant de la simplicité envers le Christ. Si le premier venu en effet prêche un autre Jésus que celui que nous avons prêché, s'il s'agit de recevoir un Esprit différent de celui que vous avez reçu, ou un Évangile différent de celui que vous avez accueilli, vous le supportez fort bien. J'estime pourtant ne le céder en rien à ces "archiapôtres". Si je ne suis qu'un profane pour la parole, pour la science, c'est autre chose; en tout et devant tous, nous vous l'avons montré.» (2Co 11,1-6)

De même qu'est menacée l'intégrité du mariage et de l'amour humain, Paul souligne combien est menacée aussi la confession de foi par un syncrétisme et un relativisme généralisé. Mais reconnaissons que cette intransigeance nous fait peur, elle peut conduire au fanatisme religieux. D'où le second aspect de la stratégie paulinienne, pour garantir l'authenticité évangélique de son intransigeance:

\section{La médiation apostolique au service de la vérité} de l'Évangile et de l'authenticité de l'amour de Dieu

J'en arrive maintenant à notre épître de référence, la première lettre aux Thessaloniciens: Paul y fait l'apologie de son apostolat. A-t-il été accusé ? Ce n'est pas certain, mais en tout cas (sans défendre son titre d'apôtre 
comme il le fait dans l'épître aux Galates et dans la plupart des lettres ensuite) il fait l'apologie de sa manière d'être et de prêcher l'évangile. Sa venue n'a pas été «vaine» (eis kenon) malgré sa fatigue et toutes sortes de persécutions. Son apostolat n'est pas inspiré de motifs humains: ni volonté de tromper, ni impureté déguisée sous des motifs religieux, ni ruse. «Seulement, Dieu nous ayant confié l'Évangile après nous avoir éprouvés, nous prêchons en conséquence, cherchant à plaire non pas aux hommes mais à Dieu qui éprouve nos cœurs. » (1Th 2,4). Un motif bien connu qui se retrouvera dans la lettre aux Galates que nous avons déjà citée. Et Paul continue son apologie: pas de mot de flatterie, ni cupidité, ni recherche de vaine gloire (v. 6), et surtout: «alors que nous pouvions, étant apôtres du Christ, vous faire sentir tout notre poids» (v. 7). Voilà notre problématique: l'Apôtre était dans son droit de faire valoir son autorité d'apôtre investi par Dieu, par le Christ glorieux, pour le service de l'Évangile. Il pouvait faire valoir cette autorité sur un double plan. Le plan économique (se faire entretenir et payer par eux) et le plan des relations humaines avec les personnes et la communauté (s'imposer à eux). C'est précisément à ce double niveau que l'Apôtre ouvre des voies originales et évangéliques:

— il a tenu à travailler pour ne pas être à charge: "Rappelez-vous, frères, notre fatigue et notre peine: travaillant jour et nuit pour ne pas être à votre charge, nous vous avons annoncé l'Évangile.» (1Th 2,9)

- mais ce choix n'est pas que financier: l'apôtre n'a pas seulement travaillé pour gagner de l'argent, mais c'est «en travaillant» qu'il a annoncé l'Évangile, dit le texte. Nous savons qu'il était fabricant de tentes, comme Prisca et Aquila. Dans sa boutique d'artisan, Paul pouvait rencontrer les gens et leur annoncer l'Évangile. Ce choix n'est pas donc pas seulement financier mais bien apostolique. Nous aurions pu nous imposer tant au plan financier qu'au plan de l'autorité, pourtant, dit Paul, nous y avons renoncé. La seconde partie du v. 7 est pour moi déterminante et éclaire tout son apostolat:

Au contraire, nous nous sommes faits tout aimables au milieu de vous. Comme une mère nourrit ses enfants et les entoure de soins, telle était notre tendresse pour vous que nous aurions voulu vous livrer en même temps que l'Évangile de Dieu, notre propre vie, tant vous nous étiez 
devenus chers. Vous vous souvenez, frères, de nos labeurs et fatigues: de nuit comme de jour, nous travaillions, pour n'être à la charge d'aucun de vous, tandis que nous vous annoncions l'Évangile de Dieu! Vous êtes témoins, et Dieu l'est aussi, combien notre attitude envers vous, les croyants, a été sainte, juste, sans reproche. Comme un père pour ses enfants, vous le savez, nous vous avons, chacun de vous, exhortés, encouragés, adjurés de mener une vie digne de Dieu qui vous appelle à son Royaume et à sa gloire. (1Th 2,7-12)

$\mathrm{Au}$ lieu de faire valoir et peser son autorité, Paul se compare à une mère et ensuite à un père. Et il donne le nom de frères à sa communauté. L'Évangile crée ainsi une atmosphère de famille: il y manque seulement le registre de l'épouse-époux que Paul mentionne dans la lettre aux Corinthiens.

Davantage encore: Paul se compare à une mère en train de nourrir son enfant, de l'entourer de soins. Combien de chrétiens sont-ils conscients que la situation de transmission de l'évangile, pour Paul, est celle d'une proximité de tendresse et d'amour, la mère tirant d'elle-même ce qu'elle donne à son enfant? Cette tendresse est d'ailleurs essentielle à l'homme qu'il sera plus tard. Mais attention: la scène n'est pas qu'émouvante. Ce qui est souligné n'est pas seulement la tendresse de l'Apôtre (Paul ne devait pas être un homme particulièrement doux) mais bien la situation de communication de l'Évangile.

«Telle était notre tendresse pour vous» écrit-il. Cette attitude de proximité et de tendresse se révèle dans l'occasion de la lettre $(1 \mathrm{Th} 2,17)$, lorsque Paul (avec Silvain et Timothée) se dit «orphelin» de la communauté de Thessalonique (aporphanisthentes: apo + orphanizô). Depuis son départ de Thessalonique, l'apôtre dit qu'ils lui ont été arrachés et qu'ils lui manquent profondément. De même que les parents manquent à des enfants orphelins, de même l'apôtre se dit orphelin d'eux. L'Évangile avait créé entre eux des liens humains profonds, de famille. Si ce type d'amitié et de proximité manque, si manque la charité, quelque chose d'aussi essentiel que l'air pour le vivant manque à la communauté chrétienne.

«Nous aurions voulu vous livrer, en même temps que l'Évangile de Dieu, notre propre vie, tant vous nous étiez devenus chers (agapètoi)» (v. 8). Dans la transmission de l'Évangile, il ne peut donc y avoir seulement le souci du contenu, de la doctrine (orthodoxie). Paraît nécessaire également la préoccupation de la médiation humaine, et d'une médiation qui 
soit crédible (orthopraxie). L'Évangile ne peut se détacher de la proximité et de l'amitié qui donnent du poids aux paroles. L'apôtre (et chaque baptisé est appelé à être apôtre) est invité à joindre à l'annonce de l'Évangile quelque chose de sa propre vie, de son amour pour le prochain. C'est cela qui va accréditer ses paroles qui, sinon, apparaîtraient comme vides... Comme le montre le début de la lettre:

"Car notre Évangile ne s'est pas présenté à vous en paroles seulement, mais en puissance, dans l'action de l'Esprit Saint, en surabondance. » (1Th 1,5a) Non pas du blabla, mais une expérience de plénitude. L'Évangile consiste dans une expérience de plénitude, bien-être à la fois du corps et de l'âme, bien-être familial en communauté ou dans la société. Notons encore l'autre nuance: «De fait, vous savez comment nous nous sommes comportés au milieu de vous pour votre service.» (1Th $1,5 \mathrm{~b})$ Le texte grec est un peu moins explicite: «au milieu de vous et pour vous». L'apôtre n'est pas au-dessus de sa communauté mais au milieu d'eux. Rappelons-nous la parole de Jésus: "Je suis au milieu de vous comme celui qui sert.» (Lc 22, 27) Il faut prendre en compte le contexte de cette déclaration de Jésus. Dans l'évangile de Luc, elle suit l'institution de l'eucharistie:

Il s'éleva aussi entre eux une contestation: lequel d'entre eux pouvait être tenu pour le plus grand? Il leur dit: "Les rois des nations dominent sur elles, et ceux qui exercent le pouvoir sur elles se font appeler Bienfaiteurs. Mais pour vous, il n'en va pas ainsi. Au contraire, que le plus grand parmi vous se comporte comme le plus jeune, et celui qui gouverne comme celui qui sert. Quel est en effet le plus grand, celui qui est à table ou celui qui sert? N'est-ce pas celui qui est à table? Et moi, je suis au milieu de vous comme celui qui sert!» (Lc 22,24-27)

On voit ici combien l'apôtre est vraiment devenu disciple du Christ, pourtant sans l'avoir jamais rencontré sur les chemins de Galilée ou à Jérusalem. Mais sa connaissance ne relève pas de la chair ou de l'histoire: c'est une connaissance intérieure du Christ en son mystère pascal, une connaissance du Christ surgie de sa rencontre au chemin de Damas et toujours maintenue vivante dans le cœur de Paul, à travers son apostolat, sa prière, sa charité apostolique. Il aurait pu faire valoir ses droits apostoliques, mais précisément il y renonce pour être témoin d'un autre type d'autorité qui lui vient du Christ lui-même. Ici Paul se révèle tout proche de Jésus en son ministère terrestre: "Venez à moi, vous tous qui peinez 
et ployez sous le fardeau, et moi je vous soulagerai. Chargez-vous de mon joug et mettez-vous à mon école, car je suis doux et humble de coeur, et vous trouverez soulagement pour vos âmes. Oui, mon joug est aisé et mon fardeau léger. »(Mt 11,28-30)

De Jésus, dont le cœur fut tellement sensible aux souffrances et à la peine des hommes, à l'apôtre Paul, il y a une ligne droite et une même logique, celle de l'Incarnation et de la vie apostolique. La parole de l'agapè vient comme naturellement sous la plume de l'apôtre dans notre lettre et dans ce contexte: "Comme une mère nourrit ses enfants et les entoure de soins, telle était notre tendresse pour vous que nous aurions voulu vous livrer, en même temps que l'Évangile de Dieu, notre propre vie, tant vous nous étiez devenus chers.» (1Th 2,7-8) Jean le dira en d'autres paroles, mais c'est toujours la même logique apostolique, qui fait le lien entre l'agapè, le don de la vie pour l'Église et la condition de l'apôtre:

Nul n'a plus grand amour que celui-ci: donner sa vie pour ses amis. Vous êtes mes amis, si vous faites ce que je vous commande. Je ne vous appelle plus serviteurs, car le serviteur ne sait pas ce que fait son maître; mais je vous appelle amis, parce que tout ce que j'ai entendu de mon Père, je vous l'ai fait connaître. Ce n'est pas vous qui m'avez choisi; mais c'est moi qui vous ai choisis et vous ai établis pour que vous alliez et portiez du fruit et que votre fruit demeure, afin que tout ce que vous demanderez au Père en mon nom, il vous le donne. Ce que je vous commande, c'est de vous aimer les uns les autres. (Jn 15,13-17)

On pourrait penser que quand l'Apôtre devient une maman pour sa communauté (et un père avec son devoir d'exhortation qui fait grandir et appelle, mais j'ai choisi de souligner surtout l'aspect maternel ici, qui semble moins habituel dans nos représentations de l'apostolat...), il court le risque d'être moins capable de rentre gloire à Dieu, à sa transcendance et à sa gloire. C'est précisément le contraire. Reprenons comme exemple le début de l'épître aux Thessaloniciens:

De fait, vous savez comment nous nous sommes comportés au milieu de vous pour votre service. Et vous vous êtes mis à nous imiter, nous et le Seigneur, en accueillant la Parole, parmi bien des tribulations, avec la joie de l'Esprit Saint: vous êtes ainsi devenus un modèle pour tous les croyants de Macédoine et d'Achaïe. De chez vous, en effet, la Parole du Seigneur a retenti, et pas seulement en Macédoine et en Achaïe, mais de tous côtés votre foi en Dieu s'est répandue, si bien que nous n'avons plus besoin d'en 
rien dire. On raconte là-bas, comment nous sommes venus chez vous, et comment vous vous êtes tournés vers Dieu, abandonnant les idoles pour servir le Dieu vivant et véritable, dans l'attente de son Fils qui viendra des cieux, qu'il a ressuscité des morts, Jésus, qui nous délivre de la colère qui vient. (1Th 1,5-10)

C'est précisément quand l'Apôtre se fait vraiment fraternel et proche, plein d'agapè dans ses relations fraternelles envers tous, c'est précisément à ce moment que les gens peuvent reconnaître que sa parole ne vient pas seulement d'une initiative humaine mais bien de Dieu. Davantage encore: ils se mettent à imiter celui qui avait choisi de ne pas se mettre en avant! Et ils l'imitent d'une manière telle que la mission se développe, au point que l'apôtre n'a plus besoin de parler. Leur vie parle dorénavant.

\section{Conclusion}

On dit souvent: " le Christ, oui, mais l'Église non ». Nous venons de voir que sans la médiation humaine, apostolique, ecclésiale, les gens n’auront pas la possibilité de découvrir le visage du Christ ni le vrai visage de Dieu: «Dieu, personne ne l'a jamais vu... » Jésus est venu pour le révéler au monde. Et nous sommes envoyés pour prolonger cette mission: "Comment l'invoquer sans d'abord croire en lui? Et comment croire sans d'abord l'entendre? Et comment entendre sans prédicateur? Et comment prêcher sans être d'abord envoyé ? Selon le mot de l'Écriture: "qu'ils sont beaux les pieds des messagers de bonnes nouvelles!" » (Rm 10,14-15)

Mais cette médiation doit à la fois avoir un air d'autorité (certitudes que recherchent bien des gens, lassés des produits de substitution) et en même temps de grande proximité et tendresse. Une autorité "pas comme les scribes... un enseignement nouveau» (voir Mc 1,22) disaient ceux qui écoutaient et voyaient Jésus. Une autorité dont Paul a trouvé le secret auprès de Jésus crucifié, parfaitement obéissant à l'amour du Père pour le monde, et pour cela même glorifié.

Ayez entre vous les mêmes sentiments qui sont dans le Christ Jésus: Lui, de condition divine, ne retint pas jalousement le rang qui l'égalait à Dieu. Mais il s'anéantit lui-même, prenant condition d'esclave, et devenant semblable aux hommes. S'étant comporté comme un homme, il s'humilia plus encore, obéissant jusqu'à la mort, et à la mort sur une croix! Aussi Dieu 
l'a-t-il exalté et lui a-t-il donné le Nom qui est au-dessus de tout nom. (Ph 2,5-9)

Oui, l'apôtre Paul a suivi le même chemin, le même abaissement. La fidélité à l'évangile ne concerne pas seulement son contenu mais aussi et surtout le mode de sa transmission. L'autorité apostolique ne peut être que sur le mode christologique. Un témoignage exigeant, fidèle, sans concession trop facile à l'esprit du temps et aux modes. Mais rappelonsnous le parcours de Paul qui, avant sa rencontre avec le Christ, se comparait volontiers aux Pharisiens, à son avantage: "ego mallon» (moi, bien plus: plus fidèle, plus pharisien, etc.)

Mais tous ces avantages dont j'étais pourvu, je les ai considérés comme un désavantage, à cause du Christ. Bien plus, désormais je considère tout comme désavantageux à cause de la supériorité de la connaissance du Christ Jésus mon Seigneur. À cause de lui j'ai accepté de tout perdre, je considère tout comme déchets, afin de gagner le Christ, et d'être trouvé en lui, n'ayant pas comme justice à moi celle qui vient de la Loi, mais celle par la foi au Christ, celle qui vient de Dieu et s'appuie sur la foi. (Ph 3,7-9)

C'est ainsi que Paul avant sa conversion était un témoin violent, un traditionaliste intégriste, persécutant les chrétiens au nom des traditions des pères! Mais ensuite il reçoit plutôt des coups de bâton et n'en donne plus. Il se fait le serviteur de tous, il renonce à faire valoir ses droits: «Je n'ai usé, moi, d'aucuns de ces droits, et je n'écris pas cela pour qu'il en soit ainsi à mon égard; plutôt mourir que de... Mon titre de gloire, personne ne le réduira à néant. » (1Co 9,14-15)

Paul peut rassembler notre problématique (fidélité et bonté) dans une même phrase: "Ce n'est pas nous que nous prêchons, mais le Christ Jésus, Seigneur; nous ne sommes, nous, que vos serviteurs à cause de Jésus. » (2Co 4,5) Ou encore: "Aussi sûrement que la vérité du Christ est en moi, ce titre de gloire ne me sera pas enlevé dans les régions de l'Achaïe. Pourquoi ? Parce que je ne vous aime pas? Dieu le sait.» (2Co 11,10) Voilà mises ensemble vérité du Christ, médiation et crédibilité apostolique. Oui, Paul est un témoin fidèle et exigeant dans sa fidélité à l'Évangile, mais on peut aussi parler d'un témoignage de l'Évangile EN LUI, c'est-à-dire sur le mode de la kénose de Jésus. J'aimerais pour terminer rappeler les paroles décisives du cardinal Journet au Concile Vatican II, alors que la droite de l'assemblée, conservatrice, avait peur que l'on abandonne les droits de la vérité (dans le cadre de la discussion 
du décret sur la liberté religieuse): "Non, nous ne sommes pas et ne serons pas infidèles à la vérité, mais nous prenons l'engagement de ne la défendre dorénavant que par les armes de la lumière».

\section{Références}

TincQ, H. (2003), «Europe: Dieu en disgrâce », Le Monde (12 juin), p. 1.

Thalmann, R. (2003), "Oui, une constitution sans Dieu », Le Monde (25 juin), p. 15.

\section{Résumé}

Est-il possible d'invoquer une vérité et d'apparaître comme un citoyen du monde ? Le fanatisme religieux semble indiquer le contraire. Or le parcours de Paul malgré l'image stéréotypée qu'on peut en avoir - nous donne l'exemple de la conviction alliée à la douceur, deux attributs ancrés dans une expérience spirituelle à deux faces: révélation du Fils et bienveillance du Père. Une lecture de la première lettre aux Thessaloniciens permet de renouveler en ce sens notre compréhension de l'apostolat de Paul.

\section{Abstract}

Is it possible to stand up for a truth and to still appear as a citizen of the world? Religious fanaticism seems to indicate the contrary. However, Paul's life journey, despite the stereotypic image we have of him, gives us a model of conviction allied with gentleness, two attributes anchored in a two-faceted spiritual experience: revelation of the Son and kindness of the Father. A reading of the first letter to the Thessalonians allows us to renew this aspect of our comprehension of the apostleship of Paul.

() Revue Théologiques 2004. Tout droit réservé. 